\title{
Parcours réflexif de la problématique des usages : une tentative de synthèse
}

Yanita Andonova

\section{(2) OpenEdition}

1 Journals

Édition électronique

URL : http://journals.openedition.org/communicationorganisation/2960

DOI : 10.4000/communicationorganisation.2960

ISSN : $1775-3546$

Éditeur

Presses universitaires de Bordeaux

Édition imprimée

Date de publication : 2 octobre 2004

ISSN : 1168-5549

Référence électronique

Yanita Andonova, "Parcours réflexif de la problématique des usages : une tentative de synthèse », Communication et organisation [En ligne], 25 | 2004, mis en ligne le 27 mars 2012, consulté le 30 avril 2019. URL : http://journals.openedition.org/communicationorganisation/2960 ; DOI : 10.4000/ communicationorganisation. 2960

Ce document a été généré automatiquement le 30 avril 2019.

(c) Presses universitaires de Bordeaux 


\title{
Parcours réflexif de la problématique des usages : une tentative de synthèse
}

\author{
Yanita Andonova
}

1 Au sein des sciences sociales les recherches centrées sur les usages ne datent pas d'hier et ne constituent pas une nouveauté en soi. Ces approches ont suscité et continuent d'engendrer une littérature foisonnante, et ont acquis une énorme popularité auprès des chercheurs depuis plus d'une décennie. Pourtant le recours systématique à la notion d'usage crée un flou conceptuel, car les chercheurs abordent cette problématique en l'employant chacun à leur manière et sans la définir de manière précise. Dans ce cas comment cerner le champ des usages? Comment articuler l'appropriation, l'utilisation, l'impact et la pratique? Est-il question des mêmes réalités, hâtivement qualifiées sous ce vocable très commode, d'études d'usages? C'est ce questionnement que nous allons tenter d'amorcer. Devant l'ampleur du sujet, nous allons dans un premier temps définir ce que nous entendons par "usages", suivre ensuite l'émergence du champ interdisciplinaire, fournir enfin un aperçu des thématiques récurrentes, afin de proposer des repères pour les analyses ultérieures.

2 Malgré la multitude des études empiriques menées dans ce cadre, il est étonnant de constater le peu de synthèses critiques de la problématique des usages ${ }^{1}$. Ces dernières sont indispensables pour l'évolution de ce champ en pleine expansion et constituent un passage obligé pour les travaux se revendiquant, de près ou de loin, en contiguiité avec le sujet.

\section{Vers une définition de l'usage : le leurre du consensus}

Débutons ce parcours réflexif par un constat, celui de l'ambiguïté, maintes fois évoquée, rarement véritablement questionnée, de la notion d'usage. Elle est principalement due d'une part à la variété des phénomènes décrits sous ce vocable et à la diversité des approches méthodologiques d'autre part $^{2}$. Interrogeons à présent cette notion, qui 
souffre d'une grande imprécision, en raison de sa généralisation et de son sens apparemment « évident ».

Si l'on tente une première approche, on constate que les chercheurs travaillant sur cette problématique, s'empressent le plus souvent de distinguer l'usage de l'utilisation, en soulignant que ces deux termes renvoient à des logiques différentes. À quel moment précis l'«utilisation" devient-elle un «usage»?. Le critère de démarcation habituellement évoqué est celui des niveaux hétéroclites d'appropriation et d'intensité dans le maniement des techniques. L'utilisation implique une action novatrice grâce à l'outil et correspond, de fait, à l'emploi plus ou moins maladroit qu'en fait tout novice. L'usage, quant à lui, nécessite d'une part stabilité et d'autre part généralité suffisante ${ }^{3}$.

5 Au terme d'usage, certains chercheurs préfèrent celui de pratique. L'approche des pratiques de communication se propose d'observer la mise en œuvre sociale des technologies de communication pour ainsi dire «in situ». Les pratiques de communication s'élaborent autour d'une double médiation, à la fois technique et sociale : technique car l'outil utilisé structure la pratique; sociale, car les formes d'usage et le sens accordé à la pratique se ressourcent dans le corps social ${ }^{4}$. Néanmoins, dans la plupart des recherches scientifiques actuelles, ces deux notions s'amalgament.

Un consensus semble s'instaurer quant à l'emploi de la notion d'usage social qui tend de plus en plus à s'imposer. Dans cette optique, l'utilisation apparaît comme une contribution provisoire, éphémère et transitoire à un processus évolutif, qui serait l'usage social d'une technique ou d'une technologie ${ }^{5}$. Ainsi, l'usage prend en compte le cadre social plus large qu'englobent les interactions entre les individus et les objets techniques, mais également "les trajectoires d'usage ", c'est-à-dire l'histoire personnelle et sociale, spécifique à chaque individu. Pierre Chambat, dans un des rares articles de synthèse sur cette problématique ${ }^{6}$, définit la notion d'usage comme une combinaison de pratiques de communication et de représentations qui leur sont associées. Selon cet auteur, travailler sur les usages revient à mettre en perspective le statut de la technique (caractère presciptif, normatif de la technique), le statut des objets (utilisation fonctionnelle ou non de la technique, utilisation en fonction du statut social, utilisation régie par des normes sociales) et le statut des pratiques (niveau de réalité sociale).

7 Le consensus existant autour de l'emploi de la notion d'usage dans les recherches scientifiques actuelles n'est pas sans poser quelques difficultés, notamment d'ordre méthodologique. Ainsi, l'usage est mobilisé en sciences sociales d'une manière très hétérogène. Certaines recherches se focalisent sur les objets techniques dont on se sert, d'autres sur l'observation des usagers. D'aucuns mobilisent des analyses discursives, d'autres des études ethnographiques ou encore des analyses sémiotiques, articulées avec une ethnologie des pratiques de communication ${ }^{7}$. Le terrain de prédilection des approches d'usages concerne essentiellement les technologies d'information et de communication (TIC), une autre expression non moins ambiguë, qui pose des problèmes quant à la définition des objets d'études et à la mise en œuvre des approches méthodologiques. Le débat épistémologique et méthodologique pourtant fondamental, dépasse la portée de ce travail. Notons toutefois que cette apparente unanimité autour de la notion d'usage reste fragile et mérite d'être questionnée en profondeur.

8 Pour notre part, nous allons nous risquer à une définition synthétique afin de circonscrire ce champ de recherché8 ${ }^{8}$. L'utilisation, serait ce processus individuel d'appropriation, qui se transforme en usage social dès l'instant où elle devient une pratique courante, 
structurée par des représentations, englobant le cadre socio-technique plus large des interactions. En somme, l'utilisation loge du côté de l'appropriation des dispositifs techniques et prend en compte par conséquent la dimension purement fonctionnelle $\mathrm{du}$ dispositif, tandis que l'usage évoque une construction sociale permanente.

Interrogeons à présent les origines des approches sur les usages et replaçons-les dans une perspective historique. Cette dernière se révèle significative quant aux tensions, oppositions, difficultés et interrogations liées à l'émergence de ce champ interdisciplinaire.

\section{Genèse du champ d'étude des usages}

Trois principaux axes de recherches ont concouru, nous semble-t-il, à la construction progressive du champ des usages et à son enracinement dans les recherches francophones et anglo-saxonnes ${ }^{9}$. Dans un premier temps, les recherches sur les médias de masse ont remis en question la place des usagers téléspectateurs et ont scruté de près la problématique de la réception. De leur côté, les travaux sur la télématique et l'autonomie sociale ont donné une épaisseur sociale à l'usage et ont mis en avant la socialité en réseau. Par la suite, les analyses se sont élargies en s'intéressant à la place des acteurs (humains et non-humains, utilisateurs, concepteurs) et de la technique dans les processus d'innovation, en apportant ainsi leur contribution à la constitution du champ des usages.

\section{La figure de l'usager-téléspectateur dans l'étude de la réception médiatique}

11 La notion d'usage des médias a fait son apparition dans les recherches scientifiques, à l'origine essentiellement anglo-saxonnes, dans les années

1270 avec le courant « usages et gratifications ». Les travaux initiés par Paul Lazarsfeld et par son équipe de l'Ecole de Columbia sont généralement considérés comme fondateurs de cette tendance, car ils ont introduit au sein des recherches préexistantes, centrées exclusivement sur les effets directs des médias, la notion d'audience active et ont insisté sur la liberté de choix du téléspectateur devant l'offre de programmes. Les chercheurs faisant partie de ce courant expriment l'hypothèse d'une diffusion par niveau (two-step flow), et non plus d'une influence directe (one-step flow) des médias sur les téléspectateurs ${ }^{10}$. Chaque usager de la télévision est susceptible d'être affecté, suivant ses besoins psychologiques et sociaux de gratification, de manière tout à fait distincte par un message télévisuel. On insiste alors sur la variété des besoins, des orientations et des activités interprétatives repérables chez les membres d'un public qui diffèrent selon leurs caractéristiques sociales et individuelles. Si le rôle du courant " usages et gratifications » n'est pas central dans l'émergence du champ des usages, on peut néanmoins lui reconnaître l'avantage d'établir la transition entre les recherches sur les effets des médias et celles orientées vers la réception.

13 Les Cultural Studies ont également contribué à approfondir la notion d'usager en étudiant la culture des sociétés modernes industrielles ${ }^{11}$. Les recherches issues de ce courant britannique se sont orientées vers le moment de la réception et ont proposé d'étudier les stratégies de décodage des messages télévisés à l'œuvre à ce moment précis ${ }^{12}$ Une des 
principales conclusions de ces travaux consiste à affirmer que les écarts existants dans l'interprétation d'un message sont causés d'abord par des facteurs sociaux et culturels constitutifs de «l'ordre culturel dominant». D'autre part, la notion de «lecture préférentielle $»^{13}$ vers laquelle le texte oriente sans cesse le lecteur s'avère, malgré ses limites, décisive pour le développement des travaux ultérieurs. Certaines analyses issues de ce courant portent sur l'articulation de la sphère publique et privée et étudient de manière détaillée le processus de décodage des messages télévisuels dans le contexte domestique, ainsi que les structures de la communication familiale au sein de laquelle s'inscrit la consommation quotidienne de la télévision ${ }^{14}$. D'autres travaux se présentent comme une critique culturelle ayant comme objectif de défier les vues dominantes sur la télévision populaire et ses publics $1^{15}$. Ainsi s'esquisse un nouveau statut du message médiatique, au sein duquel l'usager-téléspectateur participe activement à la construction du sens. L'élément déterminant devient l'interaction entre les contenus médiatiques et les lectures qui en sont faites par les usagers. Le concept de "lecture » succède peu à peu à celui de "gratification » du contenu des productions de la culture de masse, qui prend en compte la pluralité des lectures, la variété des interprétations propres à chaque individu. Cette approche présente également des limites, la principale étant la tendance de définir les publics comme étant structurés principalement par la réalité sociale. Ainsi ce modèle s'avère lui-aussi incomplet et la nécessité de penser autrement la réception médiatique s'impose.

Les études centrées sur l'usager se sont enrichies, au cours des années 80-90, avec les travaux sur la réception de la télévision dans le contexte familial quotidien. Il s'agit de l'observation des sujets en "milieu naturel ", centrées non plus sur les interactions sémantiques entre les téléspectateurs et les textes médiatiques, mais sur l'appréhension des usages médiatiques ${ }^{16}$. On s'intéresse davantage aux styles de relation des téléspectateurs face à la réception télévisuelle ${ }^{17}$. Ces recherches mobilisent des techniques ethnographiques, afin de récolter le maximum d'éléments significatifs pour décrire finement les pratiques quotidiennes de réception médiatique ${ }^{18}$.

Malgré les impasses et les multiples critiques, le courant d'études sur la réception ${ }^{19} \mathrm{a}$ profondément marqué les travaux de recherche ultérieurs et a contribué aux acquis des sciences sociales, notamment sur deux plans : celui du statut de l'usager des médias, qui dorénavant sera considéré comme un acteur dans l'élaboration du sens des messages qu'il reçoit et non plus comme un être passif, simple consommateur d'idéologie dominante, et celui du contenu de ces messages, qui se prête à diverses interprétations.

\section{La problématique de « l'autonomie sociale » dans l'usage de la télématique et de la micro-informatique}

Une impulsion importante aux recherches sur les usages a été donnée par la démocratisation du micro-ordinateur et par le développement des techniques numériques. La multiplication des chaînes de télévision et les nouveaux services intégrant informatique, télévision et audiovisuel a progressivement déplacé le centre d'intérêt des chercheurs vers l'individualisation des outils de communication. L'usage de ces dispositifs est apparu comme favorisant l'initiative et la liberté individuelle et comme l'unique moyen de réduire le poids des médias de masse, d'échapper à la tyrannie de la télévision. Avec la fonction messagerie du Minitel, l'interactivité de la machine est devenue réalité et la communication médiatisée homme-machine s'est progressivement 
banalisée. Les acquis des recherches sur l'autonomie sociale, identifiant le rôle premier de l'usager dans la définition de ses pratiques, s'avèrent également très précieux pour aborder la problématique des usages. Comme nous l'avons mentionné, depuis les années 70 l'émergence de l'usager "actif et autonome» est devenue figure courante de l'évolution des systèmes d'information. Dans le cas de l'usage de l'ordinateur personnel, l'autonomie est recherchée à travers la maîtrise de la technique, source d'un accomplissement personnel. Dans le cas d'usage de la messagerie conviviale, elle permet la construction d'espaces autonomes de communication électronique hors des interdits sociaux.

De cette époque fertile en projets, date la publication d'un ouvrage sur l'usage social du magnétoscope $\mathrm{e}^{20}$, qui sera considéré comme précurseur de l'approche francophone de la sociologie des usages. Une des grandes spécificités de cette dernière en France, c'est qu'elle s'est très rapidement orientée vers les technologies d'information et de communication (TIC), en reconnaissant le rôle actif de l'usager dans l'emploi de la technique et en rejetant la perspective techniciste ${ }^{21}$. Néanmoins les premiers travaux de la sociologie des usages se sont essentiellement centrés sur les thématiques de l'autonomie sociale, de la montée de l'individualisme ${ }^{22}$ et de la sociologie de la famille ${ }^{23}$.

Pour achever ce bref tour d'horizon, nous pouvons résumer qu'initialement les recherches françaises se sont intéressées à l'usage social du vidéotex et du magnétoscope dans le milieu domestique et se sont ensuite propagées dans le domaine de l'entreprise. Les travaux ont rapidement recouvert l'analyse d'autres machines à communiquer comme le téléphone mobile, le répondeur, le cédérom, l'Internet. De manière concomitante, le courant de l'autonomie sociale et la démocratisation des systèmes de communication ont contribué à l'engouement des chercheurs pour les usages des TIC et au regain d'intérêt pour les usagers, dont le statut demeure pourtant toujours ambigu.

\section{La notion d'acteurs dans la sociologie de l'innovation}

Les travaux issus de la sociologie de l'innovation ont renouvelé le regard porté sur les usagers en observant la réalisation ou l'irréalisation des projets techniques. Cette sociologie de la traduction ${ }^{24}$, par opposition à la sociologie des sciences et des techniques classique $^{25}$, a retenu essentiellement l'usage au niveau de la conception de l'objet sociotechnique sans privilégier pour autant le champ de la communication. Elle a contribué à la montée en puissance de l'approche des usages essentiellement grâce au questionnement sur les liens qu'entretiennent technique et société dans la réalisation des objets techniques. La notion d'acteurs, humains et non-humains, qui émane de cette approche, malgré les critiques qu'elle a occasionnées, paraît profitable à l'avancement des analyses du champ, car elle a fait découvrir peu à peu le rôle important joué par les usagers dans le processus d'innovation et dans la conception même des objets techniques. L'acteur (utilisateur, concepteur, machine ou artefact) est appréhendé non pas directement en tant qu'usager, mais en tant que quelqu'un qui a un rôle à jouer avec un statut actif ${ }^{26}$. Cependant ces travaux ont suscité des réactions contrastées: d'aucuns les ont accueillis comme constituant un apport majeur à la sociologie des sciences et des techniques, d'autres n'ont pas manqué de souligner, parfois avec insistance, leurs limites et leurs faiblesses ${ }^{27}$. Néanmoins leur contribution semble importante pour la remise en question de la problématique de l'utilisateur des objets techniques, notamment en tant qu'acteur, faisant partie du processus d'innovation ${ }^{28}$. 
Chemin faisant, la notion d'usage a pris une nouvelle densité et le champ d'études des usages s'est peu à peu constitué. Malgré les parentés existantes, les acquis des recherches sur la réception, la télématique et l'innovation ne peuvent pas être transférés tels quels dans le champ des usages, mais méritent d'être révisés, contextualisés et repensés.

\section{Approches transversales et perspectives thématiques}

Dresser un panorama complet des recherches d'usages et des recherches sur les usages en proposant une grille de lecture homogène d'un domaine aussi éclaté, parait utopique et ne constitue pas notre objectif principal. Il semble néanmoins possible d'identifier quelques grandes thématiques, souvent convoquées dans ces études, afin de fournir une lecture critique des différents courants de recherche et des principales problématiques. Nous proposons d'organiser l'état des lieux des recherches sur les usages à partir de six grands chantiers transversaux, tout en insistant sur le fait que ces problématiques s'entrecroisent inlassablement au sein d'études multiples et variées.

\section{Généalogie et filiation des usages}

Certains travaux issus de ce champ interdisciplinaire rappellent les parentés existantes entre les objets techniques et leur continuité en rapport avec les usages. Les outils techniques se multiplient et se complètent, les usages se diversifient et sont souvent le prolongement de pratiques sociales déjà pré-établies. Une filiation s'effectue entre l'emploi des anciens et des nouveaux outils de communication ${ }^{29}$. Les phases d'adoption, de découverte, d'apprentissage et de banalisation participent à l'inscription sociale des $\mathrm{TIC}^{30}$. Ainsi la généalogie des machines à communiquer (lanterne, radio, télévision, téléphone, l'appareil photo, vidéo) peut apporter des enseignements utiles sur l'évolutivité des usages ${ }^{31}$. Une remontée dans le temps parait profitable pour ces analyses, qui ont souvent tendance à oublier la filiation et la complémentarité des techniques, afin d'insister sur le fait que l'usage exige un long processus d'appropriation, qu'il n'est jamais immédiat et qu'il possède parfois, comme pour le téléphone, une longue histoire sociale.

Le temps technique est-il autonome par rapport au temps des usages? L'approche sociotechnique insiste sur l'usage social des techniques dans le temps et sur la construction collective de la technique et du social. Il est désormais inconcevable d'analyser les choix technologiques en dehors de leur cadre historique respectif, car le concepteur se représente toujours l'usage de l'objet technique qu'il élabore ${ }^{32}$ et lui confère un projet d'usage. Par ailleurs, les concepteurs sont le plus souvent les premiers utilisateurs des artefacts qu'ils ont créés ${ }^{33}$. Ainsi les études issues de ce premier axe de recherches apportent une contribution importante à l'évolution de la problématique des usages.

\section{Processus et dynamiques d'appropriation}

Une grande partie des premiers travaux sur les usages sont consacrés à l'analyse de l'insertion des objets techniques en terme d'appropriation et d'impact. Nous avons déjà mentionné que les notions d'utilisation, d'usage et de pratique au sein des recherches actuelles corrèlent des niveaux différents d'appropriation. On a vu ainsi apparaître le souci de mettre l'accent sur la dimension dynamique de l'appropriation d'une 
technologie. Dans ce processus, la banalisation de la technique joue un rôle considérable. Celle-ci ne consiste pas seulement à rendre la complexité plus accessible en simplifiant l'emploi de l'objet, mais parait essentielle pour que s'opère une appropriation qui transforme l'objet technique en outil social. Des études se proposent d'observer les « usagers ordinaires » dans la période d'émergence de l'Internet grand public en mettant en lumière «la réinvention constante des techniques qui restent la condition de leur survie $»^{34}$.

D'autres recherches ont privilégié la construction de l'identité personnelle et de l'identité sociale des individus dans les processus d'appropriation des TIC. La réussite de la télématique a pu être expliquée en partie grâce à la quête identitaire qu'elle autorisait ${ }^{35}$. On a également démontré que les individus s'approprient la messagerie télématique au fur et à mesure qu'ils la maîtrisent et qu'elle répond à l'image d'eux-mêmes ${ }^{36}$. Malgré le processus identitaire ambivalent, la marge de liberté, la souplesse et l'autonomie permises par l'emploi de pseudonymes et de jeux de rôles dans les messageries-dialogues, ont contribué à l'affirmation ou plutôt à la réaffirmation de l'identité propre de chaque messageur au sein du groupe ${ }^{37}$. Dans la même optique, d'autres chercheurs ont mis en évidence la construction de l'identité électronique, à la fois identité personnelle et sociale, dans la création d'un espace commun d'intercompréhension sur Internet ${ }^{38}$

Les processus d'appropriation des TIC en milieu professionnel constituent un autre axe prioritaire d'études. Ceux-ci s'avèrent profondément hétéroclites selon les métiers ${ }^{39}$ et permettent une remise en question des identités professionnelles. L'appropriation des TIC dans les entreprises débute le plus souvent par un phénomène de substitution avec les médias existants, qui n'est pourtant que partielle. On observe ensuite un repositionnement des outils de communication les uns par rapport aux autres. Des chercheurs ont également articulé le concept d'appropriation proposé par la sociologie des usages et la théorie de la régulation conjointe, en concluant que la culture de métier et la proximité apparaissant comme des éléments déterminants des formes d'appropriation collective de l'informatique ${ }^{40}$.

Le concept d'appropriation, souvent convoqué par la problématique de l'autonomie sociale, est intéressant dans la mesure où il met en valeur le rôle productif de l'usager. Cette approche peut toutefois conduire à surévaluer l'autonomie de l'usager, à sousestimer les conditions sociales et culturelles des pratiques, et à négliger les qualités intrinsèques des objets à approprier.

\section{Résistances, détournements et bricolages d'usages}

La marge de manœuvre des usagers dans l'appropriation d'un média résiderait dans sa capacité de détournement, de mésusage ou de rejet. Cette problématique, qui constitue un autre axe important des recherches sur les usages, s'est inspirée des travaux fondateurs de Michel de Certeau, et plus particulièrement de son interrogation sur les pratiques quotidiennes ${ }^{41}$. Cet auteur dont l'influence a été considérable-sur les travaux du champ, a analysé l'écart entre les usages inventés et des usages constatés en affirmant l'existence de deux mondes qui participent à l'invention du quotidien: celui de la production et celui de la consommation (ou des usages). Ce dernier est entendu comme une production rusée, une "poḯtique », une activité de "bricolage » et de "création ». Ainsi l'usage devient une technique, une ruse et un art du faible. L'utilisateur-lecteur, supposé au préalable voué à la passivité et à la discipline, devient ainsi un inventeur 
méconnu, un producteur silencieux. Les études sur les usages se sont alors orientées vers les problématiques de détournements et d'invention d'usages, notamment des TIC. Les gens n'adoptent pas simplement une technologie, ils l'adaptent à leurs besoins dans des situations déterminées. Ils la modèlent, l'ajustent, la re-créent, parfois la réinventent. Les analyses proposées par de Certeau ont été très discutées. On lui a reproché de faire preuve d'un déterminisme social, car il ne s'intéressait qu'aux « tactiques » des pratiques sans tenir compte des «stratégies ». Le plus grand danger, nous semble-t-il, est le mythe de la résistance populaire qui induit une vision réductrice de la complexité sociale, car elle conduirait à deux réactions possibles face à l'introduction des TIC : l'adoption passive ou la résistance. Néanmoins des études de référence ${ }^{42}$ sur cette problématique de la résistance au changement ont démontré que la réalité est bien plus complexe qu'elle ne paraît en première analyse.

\section{Significations d'usage, représentations et lien social}

En étudiant les processus de médiation dans la diffusion des technologies d'information et de communication, certains chercheurs se sont attachés à préciser la place qu'occupent les machines à communiquer dans la vie quotidienne ${ }^{43}$ Ces auteurs, en identifiant les significations explicites et latentes dans lesquelles les utilisateurs eux-mêmes construisent leur usage, ont proposé une grille d'analyse sociologique. Ainsi, les significations d'usage qui confèrent aux machines à communiquer leur place sociale, sont tout aussi importantes dans l'insertion sociale des TIC que les qualités intrinsèques de ces dispositifs.

Une autre frange de chercheurs insiste sur le poids des représentations, spécifiques pour chaque individu et constitutives de sa pratique, dans l'usage des technologies. Dans le cadre de la socio-politique des usages ${ }^{44}$, une analyse en ternie de représentations parait féconde puisqu'elle permet de prendre en compte, les dimensions politique et symbolique, souvent négligées dans les usages au dépens de la dimension économique qui fait l'objet de nombreuses études. Cette approche s'intéresse également à la manière dont les concepteurs tentent d'agir sur les représentations que se font les utilisateurs de la technologie, afin d'orienter leur usage selon leurs propres objectifs. D'autres chercheurs ont démontré que les représentations des usagers vis-à-vis des cédéroms culturels diffèrent selon le contexte d'usage, mais également selon le « projet d'usage » de chaque utilisateur ${ }^{45}$.

Quant au lien social et aux formes de sociabilité permises dans le rapport médiatisé, la sociologie dispose d'un héritage long et conséquent ${ }^{46}$. La majorité des auteurs qui se sont intéressés à ce sujet constatent que l'usage des TIC ne remet pas forcément en cause les formes ordinaires de sociabilité et observent une transformation des processus de reconnaissance sociale. On échappe à certains types de relations sociales, comme par exemple la messagerie télématique qui se révèle à la fois un simulacre d'espace public et où l'anonymat n'est qu'un « simulacre d'anonymat » dans le dévoilement de l'identitée ${ }^{47}$.

\section{Rapports sociaux et formes organisationnelles}

Les approches d'usages touchent de près une autre problématique non-négligeable, celle des rapports sociaux de pouvoir. Que ce soit au sein de la vie privée ou de la vie 
professionnelle, l'usage des TIC redéfinit les liens existants entre les êtres humains. Les travaux sur le téléphone mobile font avancer avec beaucoup de discernement la problématique de l'autonomie qui pèse sur les travailleurs nomades ${ }^{48}$. L'arrivée des TIC dans la sphère domestique réaménage les relations au sein de la famille, où la frontière entre vies privée et professionnelle s'estompe. Ceci peut être vécu comme l'acquisition d'une certaine indépendance, mais aussi comme un isolement. On observe alors une déconstruction des espaces public et privé ${ }^{49}$.

Des études en milieu professionnel ont démontré que l'usage des TIC affecte inéluctablement les structures organisationnelles, les identités professionnelles et la distribution du pouvoir ${ }^{50}$. Les technologies participent à cette réorganisation de la nature du travail, qui se traduit par une flexibilité accrue et une individualisation des tâches ${ }^{51}$ et permettent également une recomposition des métiers et des compétences. Facteurs d'autonomie pour les uns, de rigidification du travail pour les autres, l'usage des TIC a suscité beaucoup d'espérances (l'exemple du télétravail est éloquent ${ }^{52}$ ), néanmoins l'observation des pratiques des entreprises a conduit à des jugements plus nuancés et à des résultats moins fructueux ${ }^{53}$.

D'autres recherches issues du champ des usages se proposent d'étudier l'évolution du Travail Collaboratif à Distance ${ }^{54}$. Comment la coopération au sein des équipes de travail se trouve-t-elle renouvelée par l'usage de nouveaux dispositifs de communication? Le simple recours aux outils groupware crée pour certains le sentiment que la coopération est déjà acquise. En réalité, les relations, les rapports sociaux et le système de pouvoir entre les acteurs se trouvent bouleversés. La transformation des réseaux sociaux préexistants devient un objet d'études digne d'intérêt, car un nouvel équilibre des forces est à rechercher. Pourtant le Travail Collaboratif à Distance semble acquis trop hâtivement, car il n'est pas toujours possible ni souhaitable d'avancer le principe de coopération si les contextes ne s'y prêtent pas ${ }^{55}$.

Dans un tout autre registre, la problématique du pouvoir est abordée en mettant en avant la question du rôle assigné aux TIC dans un contexte où se jouent des rapports de force. On parle alors de la redistribution, de l'affaiblissement et de la suppression du pouvoir des intermédiaires comme enjeux de type socio-politique ${ }^{56}$. D'autres recherches s'intéressent à la figure de l'usager en tant que citoyen et client et tentent de reconnaître leur pouvoir, même s'il s'agit d'un pouvoir contraint et fortement limité ${ }^{57}$. L'usage apparaît au croisement de trois logiques : une logique technique, qui détermine le champ des possibles, une logique économique, qui détermine le champ des utilisations rentables et une logique sociale, qui détermine la position particulière du consommateur à l'égard de ses besoins et de ses désirs ${ }^{58}$.

\section{Contexte et cadre d'usage}

Un autre type d'analyses issues du champ des usages s'est également développé, en s'attachant à étudier l'usage en tant qu'activité située ${ }^{59}$. Cette approche invite à repenser la dimension cognitive dans les pratiques avec des artefacts informationnels. L'usage et le contexte d'usage sont des éléments qui s'élaborent et se déterminent mutuellement car, selon ces chercheurs, l'environnement dans lequel se déroulent les pratiques peut être considéré comme le prolongement des capacités cognitives des êtres humains ${ }^{60}$ Dans ce cadre, l'usage est saisi comme s'insérant dans un environnement cognitif constitué de 
ressources organisationnelles structurantes, c'est-à-dire constitué en tant que réseau d'agents cognitifs humains et non-humains dans lequel il se structure ${ }^{61}$.

D'autres recherches issues essentiellement des SIC appréhendent également le contexte d'usage, mais sous un angle différent. Au sein de ces dernières, le contexte détermine le cadrage des usages dans des pratiques sociales individuelles ou professionnelles. Les usagers de leur côté, peuvent également mobiliser, dans une certaine mesure, les contextes d'usage, notamment en mettant en visibilité le sens des lieux et des situations ou tout simplement les objets techniques (TIC) ${ }^{62}$ Les environnements dans lesquels s'insère l'usager devenu co-constructeur, sont parties prenantes de l'usage. Cependant, même si les contextes sont nombreux et variés, les rapports entre usagers et contextes aboutissent à des configurations temporaires, plus ou moins durables. Ainsi, la compréhension des usages étant généralement dépendante du contexte, l'analyse de celui-ci demeure une nécessité fondamentale.

La fragilité de l'environnement cognitif partagé a également été démontrée de façon détaillée dans des recherches sur l'interaction visiophonique ${ }^{63}$ En examinant celle-ci dans le cadre d'une enquête sur le réseau expérimental de Biarritz, on a attentivement observé la manière dont s'opère une double transformation: celle d'un objet technique (le visiophone) devenu un artefact interactionnel et celle de l'interaction focalisée devenue une interaction médiatisée par l'objet technique. L'originalité de la communication visiophonique réside dans le fait qu'elle n'est pas le simple prolongement du téléphone, mais un territoire interactionnel commun. Une décennie plus tard on retrouvera beaucoup de points communs entre l'usage du visiophone et celui de la visioconférence. Les utilisateurs peuvent certes négocier avec le dispositif, chercher à le détourner ou à y introduire des éléments particuliers, sans pour autant évacuer le cadre même qu'on leur propose.

\section{Lacunes, critiques et perspectives des recherches sur les usages}

Après avoir esquissé un aperçu des questions discutées par les recherches en cours issues du champ des usages, examinons à présent les lacunes existantes et les principaux reproches, qui leur sont adressés. C'est un terrain légitime de réflexion et d'études, qui mérite un plus grand intérêt et doit être abordé avec un certain recul critique.

Les études d'usage, comme nous l'avons constaté, se sont progressivement orientées vers la prise en compte croissante de la nature complexe des processus d'usage des TIC plutôt que vers les résultats de l'appropriation. Désormais elles cherchent à comprendre ces processus de médiation et sont par leur nature-même interprétatives plutôt qu'explicatives. Toutefois quelques grandes questions restent en suspens. Elles témoignent des préoccupations qu'il faut prendre en compte dans l'analyse des usages des TIC. On en retiendra ici que trois.

41 Une première question, majeure, doit être posée. La problématique des usages est-elle suffisante pour l'analyse des pratiques avec les TIC ? Celle-ci a parfois été considérée comme défectueuse pour observer les processus de médiation avec les TIC, parce qu'elle resterait dépendante de la supposition que les objets et les techniques sont en complète extériorité par rapport aux pratiques humaines ${ }^{64}$. Pour dépasser ces critiques légitimes et saisir pertinemment la complexité grandissante des phénomènes de médiation avec les 
TIC, la problématique des usages devrait, nous semble-t-il prendre en compte trois principales dimensions : celles des discours, des pratiques et des qualités intrinsèques de la technique, au sein d'une même approche globale. Mais comment alors intégrer, assembler et agencer ces trots facteurs au sein d'études d'usage aussi hétérogènes et multiples?

En deuxième lieu, notons que certains chercheurs déplorent la montée de l'empirisme des études d'usage et de l'approche communicationnelle ${ }^{65}$. Cette constatation remet à l'ordre $\mathrm{du}$ jour une question ancienne: celle du rôle des études de cas et de leur conceptualisation. Jusqu'à quel point peut-on tenter de théoriser ces pratiques ? Doit-on encourager à l'infini l'expansion des études monographiques? Ce questionnement ne saurait réduire le rôle des études empiriques, qui s'avèrent d'une utilité heuristique afin de fournir aux scientifiques un ensemble de faits à analyser, ainsi que des instruments aptes à les évaluer, et mérite d'être approfondi.

Une dernière question concerne le manque d'études d'usages des TIC dans les métiers de production. La majorité des travaux scientifiques portant sur cette problématique concernent soit le cadre domestique familial, soit les usages professionnels de catégories socioprofessionnelles restreintes, telles les cadres et les administratifs. Comment expliquer cette indifférence des chercheurs pour les études d'usages sur des opérateurs? Il devient urgent, nous semble-t-il, d'effectuer des analyses en milieu industriel pour apporter des éléments de compréhension quant aux relations technologies - travail.

Voilà ce qui ouvre bien des pistes de réflexion pour les études ultérieures et peut inspirer les chercheurs à élargir et à approfondir la recherche sur les usages.

AKRICH M., «Les formes de la médiation technique », in Réseaux, n 60, 1993, pp. 87-98.

BABOULIN J.C., GAUDIN J.P., MALLEIN P., Le magnétoscope au quotidien. Un demi-pouce de liberté, Aubier Montaigne, Paris, 1983.

BARDINI TH., "Changement et réseaux socio-techniques : de l'inscription à l'affordance ", in Réseaux, n 76, 1996, pp. 126-155.

BEAUDOIN V., VELKOVSKA J., «Constitution d'un espace de communication sur Internet », in Réseaux, n 97, 1999, pp. 121-177.

BOULLIER D., « Les styles de relation à la télévision », in Réseaux, numéro Hors Série, 1991, pp. 119-142.

BOULLIER D., CHARTIER C, « A chacun son internet. Enquête sur les usagers ordinaires », Réseaux, n 86, 1997, pp. 159-181.

BOURDIEU P., Un art moyen : essai sur les usages sociaux de la photographie, Les Editions de Minuit, Paris, 1965.

BRUNSDON C, MORLEY D., Everyday Television: Nationwide, London, British Film Institute, 1978.

53 CERTEAU M. (DE), L'invention du quotidien. I. arts de faire, Gallimard, 1990.

CHAMBAT P. (sous la dir.), Communication et lien social. Usages des machines à communiquer Editions Descartes, Paris, 1992.

CHAMBAT P., "NTIC et représentations des usagers ", in Vitalis André (sous la dir.), Médias et nouvelles technologies. Pour une socio-politique des usages, Éditions Apogée, Rennes, 1994, pp. 45-59. 
CHAMBAT P., «Usages des TIC: évolution des problématiques », in Technologies de l'information et société, vol. 6, n 3, 1994, pp. 249-269.

CHARON J-M., «Teletel, de l'interactivité homme/machine à la communication médiatisée ", in Marchand M. et le SPES, Les paradis informationnels. Du Minitel aux services de communication du futur, Masson, Paris, 1987, pp. 95-128.

DAVALLON J., LE MAREC J., « L'usage en son contexte. Sur les usages des interactifs et des cédéroms des musées ", in Réseaux, n 101, 2000, pp. 173-195.

FLICHY P., L'innovation technique. Récents développements en sciences sociales. Vers une nouvelle théorie de l'innovation, Paris, La Découverte, 1995.

FORNEL M. (DE), « Le cadre interactionnel de l'échange visiophonique », in Réseaux, $\mathrm{n}^{\circ}$ 64, 1994, pp. 107-132.

HALL S., « Codage/décodage », in Réseaux, nº 68, 1994, pp. 27-39.

HEART PH., «Les arts de lire le réseau? Un cas d'innovation technologique et ses usages au quotidien dans les sciences ", in Réseaux, n 77, 1996, pp. 85-113.

HERT PH., «Quasi-oralité de l'écriture électronique et sentiment de communauté dans les débats scientifiques en ligne », in Réseaux, n 97, 1999, pp. 211-259.

JEANNERET Y., SOUCHIER E., «La communication médiatisée est-elle « usage »? », in Communication § Langage, $\mathrm{n}^{\circ} 132,2002$, pp. 5-27.

JOUET J., «Pratiques de communication et figures de la médiation », in Réseaux, $\mathrm{n}^{\circ}$ 60, 1993, pp. 99-120.

JOUET J., "Retour critique sur la sociologie des usages », in Réseaux, $\mathrm{n}^{\circ} 100,2000$, pp. 487-521.

JOUET J., « Une communauté télématique : les axiens », in Réseaux, n 38, 1989, pp. 49-66.

LACROIX J.-G., MÖEGLIN P., TREMPLAY G., «Usages de la notion d'usages ", in Les nouveaux espaces de l'information et de la communication, Actes du $8^{\text {eme }}$ Congrès National des Sciences de l'Information et de la Communication, 21-22-23 mai 1992, Lille, pp. 239-248.

LATOUR B., Aramis ou l'amour des techniques, Editions La Découverte, collection anthropologie des sciences et des techniques, Paris, 1993.

LATOUR B., La science en action, Éditions La Découverte, Paris, 1989.

LAULAN A.-M., La résistance aux systèmes d'information, Éditions Retz, Paris, 1985.

LE COADIC Y. F., Usages et usagers de l'information, Nathan/HER, 2001.

MALLEIN PH., TOUSSAINT Y., « Diffusion, médiation, usages des TIC », in Culture technique, $\mathrm{n}^{\circ} 24$, février 1992, pp. 219-226

44 MALLEIN PH., TOUSSAINT Y., ZAMPONI P., Les processus de médiations dans les nouvelles technologies de communication: deux études de cas, Grenoble, Université des sciences sociales/Centre d'études des pratiques sociales, 1987.

MORLEY D., Télévision, audiences\&cultural studics, London, Comedia/Routledge, 1992.

PERRIAULT J., La logique de l'usage. Essai sur les machines à communiquer, Flammarion, 1989.

PROUX S. (sous la dir.), Accusé de réception. Le téléspectateur construit par les sciences sociales, L'Harmattan, Paris, 1998.

PROUX S., « Usages des technologies d'information et de communication : reconsidérer le champ d'étude?", in Emergences et continuité dans les recherches en information cl 
communication. Actes du XII ${ }^{e}$ Congrès national des sciences de l'information et de la communication, Unesco, Paris, 10-13 janvier 2001, pp. 57-66.

QUERE L., « La situation toujours négligée ? », in Réseaux, n 85, 1997, pp. 163-192.

QUERE L., «Les boites noires de Bruno Latour ou le bien social dans la machine », in Réseaux, n³6, 1989, pp. 95-117.

SOUCHIER E., «L'écrit d'écran: pratiques d'écriture et informatique », in Connu unication\&Langagc, $\mathrm{n}^{\circ}$ 107, 1996.

TOUSSAINT Y., "Historique des usages de la télématique », in Chambat P. (sous la dir.), Communication et lien social. Usages des machines à communiquer, Éditions Descartes, Paris, 1992, pp. 193-212.

TOUSSAINT Y., "Voile et simulacre sur les messageries », in Réseaux, n 38, 1989, pp. 67-79.

VEDEL T., « Introduction à une socio-politique des usages », in Vitahs André (sous la dir.), Médias et nouvelles technologies. Pour une socio-politiquedes usages, Éditions Apogée, Rennes, 1994, pp. 13-34.

VITALIS A. (sous la dir.), Médias et nouvelles technologies. Pour une socio-politique des usages, Editions Apogée, Rennes, 1994.

\section{NOTES}

1. Les références synthétiques dans ce domaine se limitent à deux principaux articles et sont exclusivement centrées sur la sociologie des usages dans la sphère domestique, lire Chambal P., Usages des technologies de l'information et de la communication: évolution des problématiques ", in Technologies de l'Information et Société, vol. 6, n 3, 1994, p. 244-27(1; et Jouet J., « Retour critique sur la sociologie des usages », in Réseaux, n 100, 2 (100, pp. 487-521.

2. Bien que ces problèmes terminologiques ci méthodologiques soient importants, il serai ! périlleux dans le cadre restreint de cet article de s'aventurer dans la presentanoli exhaustive des définitions et des approches méthodologiques rencontrées.

3. Kouloumdjian $M-F$, Chartier $M$, «Gérer les contraintes du temps et d'espace au niveau international », in Communication et Nouvelles technologies, PPSH, 1991. p. 355.

4. Jouet J., Pratiques de communication et ligures de la médiation ", in Réseaux, n 60, 1993, pp. 99-120-160

5. Lacroix J.-C., Moëglin P., Tremplay G., « Usages de la notion d'usages », in Actes du $8^{\text {ème }}$ Congres National des Sciences de l'Information et de la Communication, 21-22-23 mai 1942, Lille, p. 244.

6. Chambat P., (1994), op.cit., p. 249-270.

7. Jeanneret Y., Souchier E., " la communication médiatisée est-elle «usage »?», in Communication Langage, $\mathrm{n}^{\circ}$ 132, juillet 2002, pp. 5-27.

8. Le propos que nous souhaitons engager ici, va au-delà de la terminologie, dont la précision est certes indispensable, mais ne constitue pas le principal objectif de cet article.

9. Par souci de clarté, nous avons choisi de présenter l'émergence de la problématique des usages d'une manière plus ou moins linéaire. Consciente qu'une telle présentation est réductrice, 
nous insistons sur le fait que plusieurs facteurs évoluent simultanément et que les phénomènes observés sont très complexes.

10. Katz-E.,Lazarsfeld P., Personal Influence, Glencoe Free Press, 1955. Lazarsfeld P.F., Berelson B., Gaudet H., The People's Choice, New York, Duell, Sloan ans Pearce, 1944.

11. Pour un bilan historique et critique de ce courant consulter le dossier thématique: les Cultural Studies, in Réseaux, n 80, novembre-décembre 199h el plus particulièrement l'article de Armand Mattelart et Éric Neveu,» Cultural Studies'Stories. La domestication d'une pensée sauvage? ", pp. 11-58.

12. Hall S.. "Encoding/decoding IV discourse ", Centre for Contemporary Cultural Studies, University of Birmingham, 1973. Hall S., « Encoding/Decoding », in S. Hall, D. Hobson, A. Lowe, P. Willis (eds). Culture, Media, Language, London : Hutchinson, 1980, pp. 128-138.

13. Corner J., "Meaning, genre \& context », in Curran J. et al (eds). Mass MediajSociety, London. Edward Arnold, 1991.

14. Morley IX, The Nationwide Audience, London, British Film Institute, 19X0 ; Morley IX, Television, audiences\$cultural studies, London, Comedia/Routledge, 1992.

15. Ang L, Watching Dallas, London, Methuen, 1945 el Aug len, « Culture de communication : pour une critique ethnographique de la consommation des médias dans le système médiatique transnationale ", in Hermès, n 11-12, 1993, pp. 75-93.

16. Voir notamment les travaux de l'américain James Lull qui portent sur les usages de la télévision au sein de la famille. Lull J., Inside Family Viewing, Routledge, London, 1990.

17. Boullier D., » Les styles de relation a la télévision ", in Réseaux, $n^{\circ} 32$, juin I9XX, pp. 119-142.

18. Proux S., «Télévision, familles et vie quotidienne: éléments d'une approche ethnographique ", in Dossier de l'audiovisuel, $n^{\circ}$ 51, La Documentation française, Paris, 1993, pp. 54-57.

19. Sur les éludes de la réception consulter $n^{\circ} 11 / 12$ de la revue Hermès : «A la recherche du public. Réception, télévision, médias ", CNRS éditions, Paris, 1993, qui propose un étal des lieux très complet de cette problématique.

20. Baboulin I.C., (laudili J.P, Mallein P.. Le magnétoscope au quotidien. Un demi-pouce de liberté, Aubier Montaigne, Paris, 1983.

21. Pour un état des lieux de la tradition francophone de la sociologie des usages cl ses spécificités par rapport aux travaux anglo-saxons, consulter l'article de Jouet Josiane, « Retour critique sur la sociologie des usages ", in Réseaux, $n^{\circ} 100,2000$, pp. 487-521.

22. Lipovelsky G., L'ère du vide. lissais sur l'individualisme contemporain, Gallimard, Paris, 1983.

23. Mallein Ph., Toussaint Y., Bydlowski M., «Télétel 3V, les adolescents et leur famille », CPE études, $\mathrm{n}$ 37.2, Ministère de la recherche, SPES-DGT, 1984.

24. Latour B., La science en action, Éditions la Découverte, Paris, 1989 ; Callon Michel, Ut Science et ses réseaux, genèse et circulation des faits scientifiques, La Découverte, Paris, 1989.

25. L'approche classique des innovations, appelées également l'approche de la diffusion, s'intéresse essentiellement de ce qui est en amont et de ce qui est en aval de chaque innovation et étudie les processus de diffusion des innovations technologiques qu'elle explique en ternie de disparités des taux d'équipement et de pratique. L'ouvrage de référence sur cette approche reste celui de Rogers 1.., Diffusion of innovations, The Free Press, New York, 1983 (3 $3^{\mathrm{eme}}$ édition).

26. Latour B., Aramis ou l'amour des techniques, éditions La Découverte, collection anthropologie des sciences et des techniques, Paris, 1993.

27. Pour une synthèse critique de cette approche se référer à Flichy P., L'innovation technique. Récents développements en sciences sociales. Vers une nouvelle théorie de l'innovation, Paris, LaDécouverte, 1993, pp. 90-109; Quéré L., «Les boites noires de Bruno Latour ou le bien social dans la machine ", in Réseaux ; n 36, juin 1989, pp.95-117 et à Boullier D., » Du bon usage d'une critique du modèle diffusionniste : discussion-prétexte des concepts de Everett M. Rogers ", in Réseaux, n³6, 1989, pp. 31-51. 
28. Akrich $\mathrm{M}$, «Les objets techniques et leurs utilisateurs. De la conception à l'action », in Conein B., Les objets dans l'action, Paris, Editions de l'EHESS.

29. Mallein Ph., Toussaint Y., «L'intégration sociale des technologies d'information et de communication: une sociologie des usages", in Technologie et société, vol. 6, n 4, 1994, pp. 315-336.

30. Sur le désenchantemcni de la technique lire loussaini Y., "Historique des usages de la télématique ", in Chambat P. (sous la dir.), Communication et lien social Usages desmachines à communiquer, Editions Descartes, Paris, 1992, pp. 123-212.

31. Periault I., La logique de l'usage. Essai sur les machines à communiquer, Flammarion, 1989.

32. Flichy P., "L'imaginaire collectif des ingénieurs : le cas des machines a communiquer ", in Réseaux, n³6, juin 1989, p.66.

33. Bardini, Horvath, «The Social Construction ot the Personal Computer User : The Risc and Fall ofthe Reflexive User -, injournal of Communication, $n^{\circ} 45$ (3), 1995, pp. 40-65.

34. Boullier D., Chartier G, » A chacun son internet. Enquête sur les usagers ordinaires », in Réseaux, n 86, novembre-décembre 1997, pp. 159-181.

35. Charon J-M., "Teletel, de l'interactivité homme/machine à la communication médiatisée », in Marchand M. et le SPES, Les paradis informationnels. Du Minitel aux services de communication du futur, Masson, Paris, 1987, pp. 95-128.

36. Poussaint Y., "Voile et simulacre sur les messageries ", in Réseaux, $\mathrm{n}^{\circ} 38$, décembre $\mid 989$, pp. 67-79.

37. Jouet J., " Une communauté télématique : les axiens ", in Réseaux, n 38, décembre 1989, pp. 49-66.

38. Beaudoin V., Velkovska |., "Constitution d'un espace de communication sui Interrici ", in Rotaia, n 97, 1999, pp. 121-177.

39. Voir Bourdieu P. (sous l.i dir.), » L'intorniaiique au travail », in Actes de la recherche en sciences sociales, $\mathrm{n}^{\circ} 134$, septembre 2000.

40. Saint Laurent A.-F., «Qui fàit quoi ? Pratiques de l'infbrmatique et résistance des métiers dans un quotidien régional ", in Acles de la Recherche en Sciences Sociales, septembre 2000, $n^{\circ} 134$. pp. 56-61.

41. Certeau M. (de), L'invention du quotidien. I. arts de faire, Gallimard, 1990.

42. Laulan A.M., ht résistance aux systèmes d'information, Editions Retz, Paris, 1985.

43. Mallein Ph.,Toussaint Y., Zamponi F., Les processus de médiations dans les nouvelles technologies de communication: deux études de cas, Grenoble, Université des sciences sociales/Centre d'études des pratiques sociales, 1987.

44. Vedel T., " Sociologie des innovations technologiques ci usagers. Introduction à une sociopolitique des usages ", in Vitalis A. (sous la dir.), Médias et nouvelles technologies. Pour une approche socio-politique des usages, Editions Apogée, Rennes, 1994.

45. Davallon I., Le Marce J, «L'usage en son contexte. Sur les usages des interactifs el des cédéroms des musées ", in Réseaux, n 101, 2000, pp. 173-195.

46. Sur la problématique du lien social, l'espace public el les formes de sociabilité, voir plus particulièrement le numéro 38, de la revue Réseaux sur les messageries, décembre 1989, ainsi que Chambat P. (sous la dir.), Communication et lien sonai Usages des machines à communiquer. Éditions Descartes, Paris. 1992.

47. Toussaint Y., "Voile et simulacre sur les messageries ", in Réseaux, n 38, décembre 1989, pp. 47-79.

48. Heurtin J-P., » La téléphonie mobile, une communication itinérante ou individuelle. Premiers éléments d'une analyse des usages en France ", in Réseaux, n90, juillet-août 1998.

49. Flichy P., (1991), Une histoire de la communication moderne espace publie et vie privée, Ed. La Découverte \& Syros, Paris ; Chambat P.. (199.S),.. Espace public, espace privé : le rôle de la 
médiation technique» in, Paillart I.(Dir.), L'espace publie et l'emprise de la communication, lui. Ellug, Grenoble, pp. 65-99.

50. Consulter à ce sujet le dossier thématique: Internet en entreprise. Réseaux, $n^{\circ} 104,2000$.

51. Francfort [., Osty F. et alii, Les mondes sociaux de l'entreprise, Desclée de Brouwer, collection «Sociologie économique », Paris, 1995.

52. Lallement M., « Une nouvelle forme d'emploi : le télétravail » in lui communication, état des savoirs, Editions Sciences Humaines, Paris, 1998, pp. 427-434 ; Breton Thierry, Le télétravail eu France. Situation actuelle, perspectives de développement et aspects juridiques, La Documentation Française, 1994.

53. Benghozi P.-J., Flichy P., Iribarne A. (d'), « Le développement des NTIC dans les entreprises françaises. Premiers constats ", in Réseaux, $n^{\circ}$ 104, 2001, pp. 31-57.

54. Notons au passage qu'en ce qui concerne celle problématique, il en existe un certain nombre de références, toutes aussi intéressantes les unes que les autres, qu'il serait impossible de citer ici.

55. Vacher B., ht gestion de l'information en entreprise: enquête sur l'oubli, l'étourderie, lit ruse et le bricolage organisés, ADBS Éditions, 1917.

56. Weissberg Jean-Louis, » Des « reality shows » aux réalités virtuelles », Terminal, n 61, 1993 ; Musso Pierre, Télécommunication et philosophie des réseaux, PUF, 1997.

57. Vedel T., Vitalis A., Orientations pour une socio-politique des usages. Rapport à l'Association Destartes, Parts, lévrier 1993.

58. Vitalis André, " La part de citoyenneté dans les usages ", in /Médias et nouvelles technologies. Pour une socio-politique des usages, Editions Apogée, Rennes, 1994, p. 38.

59. Thévenot I.., "Essai sur les objets usuels : propriétés, fondions et usages ", in Conein B., Dodier N., Thévenol I... Les objets dans l'action, l'EHESS, 1993 ; Akrich M., « Les objets techniques ci leurs utilisations. De la conception à l'action », in Conein B., Dodier N.. Thévenot I... Les objets dans l'action, l'EHESS, 1993; Hutchins P., "(Comment le "cockpit» se souvient de ses vitesses", Sociologie du travail, XXXVI, 4/94.

60. Sur la « cognition distribuée » consulter le dossier : Travail et cognition, Sociologie du Travail, XXXVI, 4/94.

61. Proux S., "Usages des technologies d'information et de communication : reconsidérer le champ d'étude?", in Émergences et continuité dans les recherches en information et communication, Actes du XII Congrès national des sciences de l'information et de la communication, Unesco, Paris, 10-13 janvier 2001, p. 62.

62. Davallon I., Le Maroc |., - L'usage en son contexte. Sur les usages des interactifs et des cédéroms des musées ", in Réseaux, n 101, 2001, pp. 173-195.

63. Fornel M. (de), «Le cadre interactionnel de l'échange visiophonique », in Réseaux, n64, p. 44, pp.107-132; Fornel M. (de), » Une situation interactionnelle négligée: la messagerie télématique », in Réseaux, n³8, décembre 1984, pp. 31-48.

64. Quéré I. - Espace public et communication. Remarques sur l'hybridation des machines el des valeurs", in Chambat P. (sous la dir.), Communication et lien social. Usages des machines a communiquer. Editions Descartes, Paris, 1992, pp. 44-45.

65. Jouet J., (2000). op.cit., pp. 487-521. 


\section{RÉSUMÉS}

Actuellement l'opinion scientifique est relativement unanime sur la valorisation des études issues de la problématique des usages. Il est par ailleurs significatif de constater combien tardive a été la prise de conscience de la synthèse du champ. Dans le cadre de cet article nous proposons d'entreprendre un aperçu des recherches menées actuellement en France sur cette problématique et d'inviter à un regard critique. Trois interrogations sont à l'origine de ce parcours réflexif : Comment peut-on définir les « usages »? Comment le champ de recherche sur les usages a-t-il progressivement émergé ? Quels sont les grands axes de contribution des études actuelles dans ce domaine? Il ne s'agit pas d'un état des lieux exhaustif des travaux dans le domaine, mais plutôt d'interrogations critiques.

Nowadays the scientific opinion on the valorization of the studies, resulting from the problems of the "uses ", is relatively unanimous. Actually, it is important to note how late the synthesis in this field has been realized. Within the framework of the present article we propose to undertake a review of the researches on these problems, carried out currently in France, and also to invite to a critical glance. Three questions are at the origin of this reflexive course: How can the " uses " he defined? How the domain of research on the uses did gradually emerge? Which arc the principal axes of contribution of the current studies in this field? It is not an exhaustive inventory of all scientific works, but rather a critical interrogation.

\section{AUTEUR}

\section{YANITA ANDONOVA}

Attachée Temporaire d'Enseignement et de Recherche (ATER) à l'Université Paris XII Val de Marne, termine actuellement une thèse en Sciences de l'Information et de la Communication au CELSA - Université Paris IV - Sorbonne, portant sur l'analyse du phénomène d'usage des TIC en milieu industriel. Domaines de compétence : communication organisationnelle; sociologie et anthropologie des usages ; analyse des techniques de communication. 